\title{
The N400 reveals how personal semantics is processed: Insights into the nature and organization of self-knowledge
}

\author{
Jason C. Coronel ${ }^{1 *}$ \\ *(Corresponding Author) \\ Assistant Professor \\ ${ }^{1}$ School of Communication \\ The Ohio State University \\ 154 N Oval Mall \\ Columbus, Ohio 43210 \\ coronel.4@osu.edu \\ Kara D. Federmeier ${ }^{2,3,4}$ \\ Professor \\ ${ }^{2}$ Department of Psychology \\ ${ }^{3}$ Beckman Institute for Advanced Science and Technology \\ ${ }^{4}$ Program in Neuroscience \\ University of Illinois \\ 603 East Daniel Street \\ Champaign, IL 61820 \\ kfederme@illinois.edu
}




\begin{abstract}
There is growing recognition that some important forms of long-term memory are difficult to classify into one of the well-studied memory subtypes. One example is personal semantics. Like the episodes that are stored as part of one's autobiography, personal semantics is linked to an individual, yet, like general semantic memory, it is detached from a specific encoding context. Access to general semantics elicits an electrophysiological response known as the N400, which has been characterized across three decades of research; surprisingly, this response has not been fully examined in the context of personal semantics. In this study, we assessed responses to congruent and incongruent statements about people's own, personal preferences. We found that access to personal preferences elicited N400 responses, with congruency effects that were similar in latency and distribution to those for general semantic statements elicited from the same participants. These results suggest that the processing of personal and general semantics share important functional and neurobiological features.
\end{abstract}

Key words: personal semantics, semantic memory, self-knowledge, N400 


\section{Introduction}

Highly detailed information can be retrieved from long-term memory, as during the recollection of autobiographical episodes. For example, someone might remember an outing with friends, including when and where the event took place and even the type of pizza that was eaten. In other cases, generalized information is used to identify objects, places, and people, and to call to mind features and relations between them that hold across contexts-for example, the fact that pizzas are often round and topped with cheese. These types of memory retrieval events have been extensively characterized in studies of episodic (for a review, see Tulving, 2002) and general semantic (for a review, see Yee, Chrysikou, \& Thompson-Schill, 2012) memory, respectively. However, there is a growing appreciation (see Renoult et al., 2012; Grilli \& Verfaellie, 2014) that some important forms of memory are intermediate in nature. One such entity is "personal semantics" (Renoult et al., 2012). Like the episodes that are stored as part of one's autobiography, personal semantics is linked to an individual, yet, like general semantic memory, it may be detached from a specific encoding context. For example, in addition to knowing that pepperoni is a popular pizza topping (general semantics), someone may know that his/her own preferred topping is mushrooms (personal semantics).

Personal semantic memory remains poorly characterized, which is surprising given its importance for cognition and everyday life. It has long been known that information is often better remembered when it is encoded with reference to the self (Rogers, Kuiper, \& Kirker, 1977), possibly because "the self is a well-developed and often-used construct that promotes elaboration and organization of encoded information" (Symons \& Johnson, 1997). However, empirical studies that examine the retrieval of information about the self have shown mixed results. Neuropsychological studies have reported intact self-knowledge in the face of 
impairments in both general semantic memory and episodic memory (Klein, Chan, \& Loftus, 1999; Klein, Cosmides, \& Costabile, 2003; Klein, Cosmides, Costabile, \& Mei, 2002), although the reverse dissociation has not been documented. fMRI studies of self-knowledge find patterns of brain activity that share commonalities with those for both general semantic and episodic memory, as well as a neural network comprised of portions of the medial prefrontal cortex (MPFC), posterior cingulate cortex, and precuneus that has been more specifically linked to selfrelated processing (for reviews, see Amodio \& Frith, 2006; Northoff \& Bermpohl, 2004; Wagner, Haxby, \& Heatherton, 2012). Some have interpreted these data to mean that self-related knowledge, and by implication personal semantic memory, is unique in terms of its functional representation in the brain (Kelley et al., 2002; Heatherton, Macrae, \& Kelley, 2004; Heatherton, 2011). Others, however, have emphasized that factors other than links to the self, such as differential demands on emotional or social processing, might instead be the root of observed behavioral and neural differences (Gillihan \& Farah, 2005; Symons \& Johnson, 1997). As Renoult et al. (2012) highlight, therefore, there is a pressing need for more research on the access of personal semantics.

Here, we provide a characterization of the retrieval of personal semantics using an electrophysiological response known as the N400, which has been fruitfully applied to the study of general semantic access for more than three decades now but has not been extensively used to assess the processing of personal semantics. In particular, we focus on a subset of what has sometimes been termed "self-knowledge", which includes a range of self-evaluative judgments about preferences, personal characteristics, and identity. The term personal semantics has also been applied to other types of knowledge, such as autobiographical facts (e.g., "My brother's name is Brian.”) or repeated events (e.g., "I pick up my brother from school every day."), which 
may rely on a distinct set of neural networks (see Renoult et al., 2015). Among types of personal semantics, self-knowledge has been argued to be most likely to be distinct from either general semantics or episodic memory (for reviews, see Renoult et al., 2012; Grilli \& Verfaellie, 2014).

Our design takes advantage of the fact that a well-studied component of the event-related potential (ERP), the N400, has been specifically linked to access of knowledge from semantic memory. The N400 is a negative-going voltage deflection that peaks around $400 \mathrm{~ms}$ after stimulus onset and has a widespread distribution with a centro-posterior maximum. It forms part of the normal electrical brain activity seen in response to a wide variety of meaningful stimuli, including visual and auditory words, faces, pictures, and sounds. Both the functional characteristics and the neural sources of the N400, primarily in medial and anterior temporal areas (for reviews, see Van Petten \& Luka, 2006; Lau, Phillips, \& Poeppel, 2008), link it to processing in semantic memory (Federmeier \& Laszlo, 2009). The amplitude of the N400 is reduced for items that are congruent with a given context (for a review, see Kutas \& Federmeier, 2011), and these N400 semantic congruency effects reflect the organization of semantic memory (Federmeier \& Kutas, 1999) and involve not only knowledge of word meanings (e.g., that trains cannot be "sour") but also world knowledge gained through experience (e.g., the fact that Dutch trains are yellow, not white; Hagoort, Hald, Bastiaansen, \& Petersson, 2004).

Our primary goal in this study is to examine the extent to which N400 congruency effects, robust and well-replicated in sentences about general knowledge (e.g., reduced N400 responses to "pepperoni" relative to "anchovies" in "A pizza topping that many people like is [pepperoni/anchovies]"), can also be obtained during the access of personal semantic information, as in "The pizza topping you like best is [artichokes/mushrooms]" (when "artichokes" are the reader's actual preference). We took care to probe personal semantics under 
conditions in which it is clearly distinguishable from general semantic knowledge. If, under these conditions, we see N400 congruency effects like those observed for access to general semantic memory, then we can conclude that there are important similarities in how personal and general semantics are accessed.

However, not all instances of meaning-based congruency affect the N400. For example, quantifiers like "often/rarely" critically change the plausibility of sentences such as, "Farmers [often/rarely] grow crops ..." but have little or no effect on N400 amplitudes to "crops" (Kounios \& Holcomb, 1992; Urbach \& Kutas, 2010). Similarly, the plausibility difference between "boys" and "eggs" as the subject of a sentence like "For breakfast, the [boys/eggs] would only eat ..." does not modulate the N400 to "eat" but instead manifests in the ERP signal as a later, posterior positivity (Kuperberg, Sitnikova, Caplan, \& Holcomb, 2003). In these cases, specific information about quantities or thematic roles does not seem to become available until after the initial access of more general semantic information (e.g., that breakfast, eggs, and eating go together).

Rather than showing up as an N400 effect then, congruency based on personal semantics might involve processes more similar to the explicit recollection of contextual details, which manifests as a late positivity, known as the Late Positive Complex (LPC) (for reviews, see Rugg \& Curran, 2007; Voss \& Paller, 2008). Enhanced late positivity (P300 or LPC) has been reported when participants are exposed to their own names or faces, as compared with other, familiar names or faces (Müller \& Kutas, 1996; Folmer \& Yingling, 1997; Tacikowski \& Nowicka, 2010), as well as in response to social vignettes presented in second (compared with third) person (Fields \& Kuperberg, 2012). These effects have been linked to greater allocation of attention to - and perhaps additional processing and evaluation of -- such self-relevant stimuli. 
In one study, for example, researchers compared ERP responses to equally famous names of politicians, celebrities, etc. that differed in that half the names were associated with high levels of personal memories (e.g., remembered watching a movie starring the celebrity of that name) while the other half were associated with low levels of personal memories (Renoult, Davidson, et al., 2015). The study found that the names elicited an N400, but its amplitude did not differ as a function of the level of personal memories associated with them. Instead, the size of the LPC was modulated by the amount of personal memories participants possessed about each person, with names linked to high levels of personal memories eliciting increased LPC amplitudes.

Here, we extended these previous studies by examining N400 congruency effects for personal semantics (as well as later effects on the LPC). We also include a general semantic condition (statements about common preferences) as a within-subject control condition in our design, allowing us to empirically examine the extent to which any N400 congruency effects obtained for personal semantics share the latency and distributional characteristics of the (wellestablished) general semantic N400 effect.

\section{Materials and Methods}

\subsection{Participants}

Sixteen University of Illinois students (seven females, mean age 22.6, age range $21-27$ ) participated for course credit or cash. Power to observe a typically-sized N400 anomaly effect with this sample size is very high (0.96). All participants, by self-report, had no prior history of psychiatric or neurological disorders and none were using psychoactive medications. All were right-handed (Oldfield, 1971). All procedures were approved by the IRB of the University of Illinois Urbana-Champaign, and all participants gave written informed consent. 


\subsection{Materials}

Materials consisted of 190 sentences regarding preferences (likes and dislikes) across a wide range of topics, including foods, drinks, sports teams, clothing and accessories, cities, countries, music, books, films, classes, holidays, chores, outings, activities, seasons, weather, animals and insects, plants, cars, games, childhood toys, electronics, relatives, politics, illnesses, and physical and personality features of significant others. Table 1 gives examples of the stimuli. Sentences were presented in either general (with reference to "many/most people", "For dessert, many people like to eat ..." ) or personal (with reference to "you", "For dessert, you like to eat ...”) form. To determine people's knowledge of general preferences, in a separate norming study, 69 University of Illinois undergraduate and graduate students $(33$ female, mean age $=$ 21.5, age range 18 - 35) were given the general sentence frames without their ending (e.g., "A topping that many people like on their pizza is ."). Participants were instructed to "write the word that first comes to mind and best finishes the sentence". Mean cloze probability (Taylor, 1953) was $46 \%$ (range $10 \%$ to $92 \%$ ).

Personal preferences were assessed for each participant in a separate session 7-10 days prior to EEG recording. Participants were given the sentence frames in personal form (e.g., "One type of sport that you like to watch is .") and asked to complete each with their preference and to rate the strength of that preference on a scale from 10 ("This is a strong, consistent preference") to 1 ("I really don't care much about this and had trouble coming up with any response"). For each preference, participants also reported what percentage of people they believed shared the same preference $(0-25 \%, 25-50 \%, 50-75 \%, 75-100 \%)$. Participants were asked to also indicate secondary and tertiary preferences for each item, and, for each, to rate the 
strength and report the percentage of people they believed would give that answer as their primary preference.

From the general norms and the personal preference assessment, we created an individualized stimulus list for each participant. Each list consisted of 25 items of four types: General Consistent (GC), General Inconsistent (GI), Personal Consistent (PC), and Personal Inconsistent (PI) (see Table 1). To ensure that responses to personal preferences could be clearly distinguished from knowledge about general preferences, we used only personal items rated high in preference $(>6)$ but low in perceived likelihood of other people sharing the preference $(\leq$ 50\%). Average personal preference strength across all participants was 9.2 and the median perceived likelihood of the preference being similar to the general population's preferences was the category " $0-25 \%$ ". For PC items, the preference provided by the individual participant was used. For PI items, the ending word was plausible, but was neither one of the participant's preferences nor given on the general preference norming. GC items had an average cloze of $70 \%$ $(\mathrm{SD}=5.8$, Range: average of $55 \%$ to $75 \%$ across participants), and GI items always had zero cloze probability ${ }^{1}$. Across the four conditions, sentences were controlled for average length (12 words). Target words were also matched across conditions for average word length (6 letters) and $\log$ frequency (.84) using the CELEX database (Baayen, Piepenbrock, \& Van Rijn, 1993).

Table 1. Sample of General and Personal Sentences that are either Consistent or Inconsistent

\section{General}

A pizza topping that many people dislike having on their pizza is anchovy/pepperoni.

A type of alcoholic drink that most people like to drink during a night out is beer/wine.

Animals that are popular as a stuffed toy are bears/snakes.

One very popular cake flavor is chocolate/banana.

A form of public transportation that many people hate using is the bus/subway.

A salad dressing that a majority of people like to put on their salad is ranch/caesar. 
One type of sport that a lot of people like to watch is football/cricket.

\section{Personal}

Your least favorite science class in high school was biology/astronomy.

Foods that you like to eat as midnight snacks are cookies/pastas.

A vegetable that you really dislike is celery/lettuce.

A type of meat that you love to eat is turkey/lamb.

A type of bread that you dislike is rye/oatmeal.

For dessert, you like to eat cheesecake/popsicles.

A genre of film you like watching is horror/fantasy.

\subsection{Procedures}

During their second session, participants' EEG was recorded as they read for comprehension. They were told that some of the sentences would be about the preferences of people in general and others about their own, personal preferences (i.e., "if the sentence says 'you' it is referring literally to you"). They were told not to blink while reading each sentence. Each trial began with fixation crosses ("++++") that were presented for a random duration between 5000 and $10000 \mathrm{~ms}$ with a total event duration of $10000 \mathrm{~ms}$. Participants were instructed to blink while looking at the fixation crosses and to not blink while reading the sentence that followed the fixation crosses. Sentences were presented word-by-word, for a duration of $250 \mathrm{~ms}$ with a $250 \mathrm{~ms}$ ISI. Sentence final words were presented for a duration of 500 ms, with 1250 ms post-sentence time before the onset of the next trial. Each word appeared in light green Helvetica 22-point font on a black background. ERPs were timelocked to the sentence final words. Sentence contexts were never repeated. For example, if a participant was shown a personal consistent sentence referring to a disliked pizza topping during the EEG study, this participant was not exposed to the general consistent/inconsistent or personal inconsistent version of this sentence during the study. 
THE N400 AND PERSONAL SEMANTICS

EEG was recorded from 26 evenly-spaced silver/silver chloride electrodes (see head icon in Figure 1) referenced online to the left mastoid and re-referenced offline to the average of the left and right mastoids; impedances were kept below $5 \mathrm{kOhms.} \mathrm{Blinks} \mathrm{and} \mathrm{eye} \mathrm{movements} \mathrm{were}$ monitored via electrodes placed on the left infraorbital ridge and the outer canthus of each eye. Electrode EEG was sampled at $250 \mathrm{~Hz}$ using Sensorium (Charlotte, VT) amplifiers set at a bandpass of 0.02-100 Hz.

ERPs were computed from $100 \mathrm{~ms}$ prior to word onset (used as a baseline) to $920 \mathrm{~ms}$ after. Epochs containing artifacts from eye movements, amplifier blocking, signal drift, or excessive muscle activity were rejected offline before averaging (average trial loss $=13 \%$ ), using detection algorithms that were separately calibrated for each participant by visual inspection. Prior to measurement, ERPs were digitally filtered with a bandpass of 0.2-20 Hz. All statistical tests are repeated measures analyses of variance (ANOVAs), with degrees of freedom adjusted by the Huynh-Feldt correction for the violation of sphericity.

\section{Results}

\section{$3.1 N 400$}

As can be seen in Figure 1, ERPs to both general and personal conditions were characterized by a prominent negativity (N400) to inconsistent conditions compared to consistent ones. N400 mean amplitudes were measured between 250 and 450 ms over all 26 channels and subjected to a three-factor repeated measures ANOVA with 2 levels of Condition (general, personal), 2 levels of Consistency (consistent, inconsistent), and 26 levels of Electrode Site. Consistent with previous studies on the $\mathrm{N} 400$, there was a main effect of Consistency, $F(1,15)=$ 49.06, $\mathrm{p}<.001, \eta^{2} p=.77$, wherein inconsistent items elicited less positive voltages $(M=2.5 \mu \mathrm{V}$ $\pm .71 S E)$ than consistent items $(M=4.7 \mu \mathrm{V} \pm .70 S E)$; this effect interacted with Electrode, 
$F(25,375)=17.01, p<.001, \eta^{2} p=.53$, displaying the typical central-posterior distribution (as assessed in detail in the distributional analysis below). There was no main effect of Condition, $F(1,15)=.83, p=.38, \eta^{2} p=.05$, nor a Condition by Consistency interaction, $F(1,15)=1.5, p=$ $.25, \eta_{p}^{2}=.09$

Given that the personal condition does elicit an N400, of primary interest for the study was to examine the extent to which the timing and distribution (as well as amplitude) of the N400 for personal preferences was similar to that for general preferences. First, to assess timing of the N400, local peak latency was obtained between 250 and 450 from the personal and general difference waves in the MiCe channel (where N400 effects are typically largest) from each participant. An additional low pass filter of $8 \mathrm{~Hz}$ was applied. We subjected the data to a one way repeated measure ANOVA with Condition (general, personal) as a factor. The latency of the N400 effect for both general $(M=358.5 \mathrm{~ms} \pm 15.2 \mathrm{~ms} \mathrm{SE})$ and personal preferences $(M=331.5$ $\mathrm{ms} \pm 15 \mathrm{~ms} \mathrm{SE}$ ) were similar (average across both is $345 \mathrm{~ms}$ ), as there was no main effect of Condition, $F(1,15)=1.5, p=.24$. The same analysis done instead over all channels (to capture the full extent of the N400 distribution) also yielded no main effect of Condition, $F(1,15)=.24$, $p=.63$, or Condition $\mathrm{x}$ Electrode interaction, $F(25,375)=.99, p=.44$.

Next, to assess the topography of the effects and further compare the size, mean voltage measures were taken in a $100 \mathrm{~ms}$ window centered around $350 \mathrm{~ms}$ (approximately the peak of the effect) from general and personal difference waves created by a point by point subtraction of consistent from inconsistent items) at 16 scalp electrodes (LLPf, LLFr, LLTe, LLOc, LMPf, LMFr, LMCe, LMOc, RLPf, RLFr, RLTe, RLOc, RMPf, RMFr, RMCe, and RMOc; see Figure 2 for schematic of electrode locations on the scalp) that were divided into factors of Hemisphere (left, right), Laterality (lateral, medial), and Anteriority (prefrontal, frontal, central, posterior). A 
THE N400 AND PERSONAL SEMANTICS

repeated measures ANOVA with Condition (general, personal) and the three distributional factors revealed significant main effects of Laterality $F(1,15)=14.38, p<.01, \eta^{2} p=.49$, and Anteriority $F(3,45)=19.78, p<.001, \eta^{2} p=.57$ and a marginal effect of Hemisphere, $F(1,15)=$ 4.7, $p=.05, \eta^{2} p=.24$. Consistent with previously reported N400 topographies (Kutas \& Federmeier, 2011), effects were bigger over medial $(M=-2.7 \mu \mathrm{V}, \pm .45 S E)$ than lateral sites $(M$ $=-1.6 \mu \mathrm{V} \pm .27 S E)$ and over the back of the head: prefrontal $(M=-.76 \mu \mathrm{V} \pm .37 S E)$, frontal $(M$ $=-1.8 \mu \mathrm{V} \pm .32 S E)$, central $(M=-2.8 \mu \mathrm{V} \pm .40 S E)$, posterior $(M=-3.35 \mu \mathrm{V} \pm .53 S E)$. Moreover, as can be seen in the topographic maps presented in Figure 3, general and personal preferences elicited similar distributions. There was a marginal effect of Condition $F(1,15)=$ $3.36, p=.09, \eta^{2} p=.18$, reflecting a tendency toward smaller effects in the personal than in the general condition. Condition did not significantly interact with any distributional variable.

\subsection{Late Positive Complex}

Although the LPC was not our main topic of investigation, and we tailored the design of the study specifically to the N400, we analyzed the LPC given previous work showing enhanced P300/LPC responses to self-relevant than non-self-relevant stimuli (Müller \& Kutas, 1996; Folmer \& Yingling, 1997; Fields \& Kuperberg, 2012; Tacikowski \& Nowicka, 2010). We measured LPC mean amplitudes between 550 and 800 ms over 15 centro-posterior channels (where LPC effects are typically largest; LMCe, RMCe, LDCe, RDCe, MiCe, MiPa, LLTe, RLTe, LDPa, RDPa, LLOc, RLOc, LMOc, RMOc, MiOc) and subjected them to a three-factor repeated measures ANOVA with 2 levels of Condition (general, personal), 2 levels of Consistency (consistent, inconsistent), and 15 Electrode sites. There was a main effect of Condition, $F(1,15)=5.52, p=.03, \eta^{2} p=.27$. Consistent with previous studies, personal 
THE N400 AND PERSONAL SEMANTICS

preferences elicited greater positive voltages $(M=6.8 \mu \mathrm{V} \pm .62 S E)$ than general preferences $(M$ $=5.84 \mu \mathrm{V} \pm .72 S E)$. There were also significant Condition x Electrode, $F(14,210)=2.8, p=$ $.03, \eta^{2} p=.16$, and Consistency x Electrode interactions, $F(14,210)=3.5, p=.02, \eta^{2} p=.19$. There was no main effect of Consistency, $F(1,15)=2.6, p=.13$, or interaction between Condition and Consistency, $F(1,15)=.05, p=.82$.

To assess the topography of the LPC response to personal (versus general) preferences, mean voltage measures were taken for personal and general statements, collapsed across consistency, within the $550 \mathrm{~ms}$ to $800 \mathrm{~ms}$ time window at the same 16 electrode sites used for the N400 topographic analysis. These were subjected to a repeated measures ANOVA with Condition (general, personal) and the three distributional factors (Hemisphere, Laterality, and Anteriority). Overall the LPC showed its typical topography, increasing in amplitude from the front to the back of the head, $F(3,45)=28.23, p<.001, \eta^{2} p=.65--$ prefrontal $(M=3.9 \mu \mathrm{V} \pm$ $.53 S E)$, frontal $(M=4.3 \mu \mathrm{V} \pm .30 S E)$, central $(M=5.5 \mu \mathrm{V} \pm .54 S E)$, posterior $(M=6.5 \mu \mathrm{V} \pm$ $.57 S E)$-- and larger over medial $(M=6.8 \mu \mathrm{V} \pm .72 S E)$ than lateral sites $(M=3.4 \mu \mathrm{V} \pm .39 S E)$, $F(1,15)=64.93, p<.001, \eta^{2} p=.81$. Although LPC responses are sometimes found to be leftlateralized, responses here were not biased (left: $M=4.9 \mu \mathrm{V} \pm .54 S E$; right: $M=5.2 \mu \mathrm{V} \pm .57$ $S E)$. There was again a main effect of Condition, $F(1,15)=5.64, p=.03, \eta^{2} p=.27$, as well as a significant interaction between Condition and Laterality, $F(1,15)=9.27, p=.008, \eta^{2} p=.38$, reflecting the fact that LPC differences between the Conditions were most salient over medial sites, where the effect was larger overall. There were no other interactions between Condition and the distributional factors. 
Figure 1. ERPs to General and Personal Items

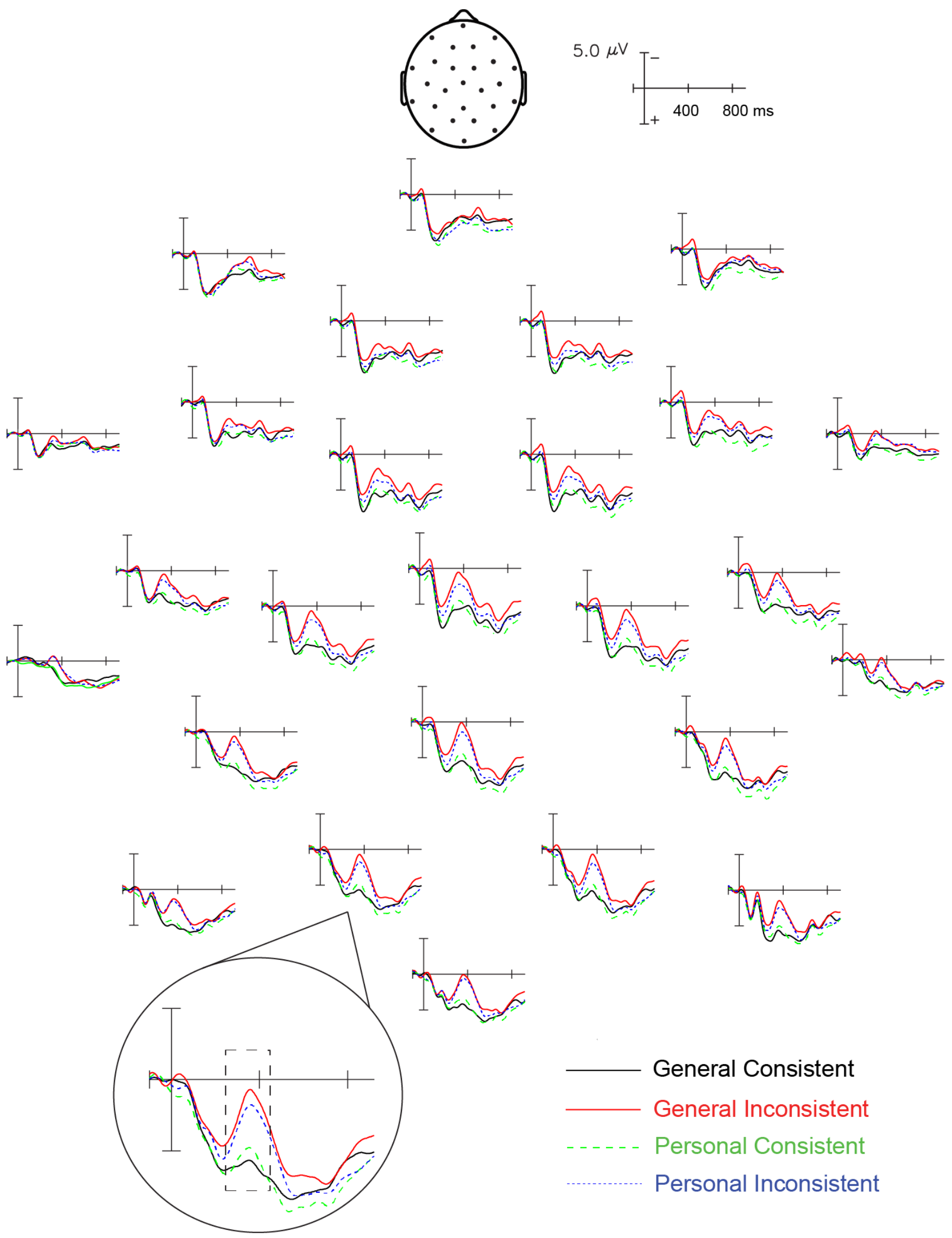

Note: Figure shows grand-average ERPs at 26 electrode sites for all four conditions. The N400 timewindow (250 $450 \mathrm{~ms}$ ) is highlighted on the magnified electrode site. 
Figure 2. General and Personal Difference Waves

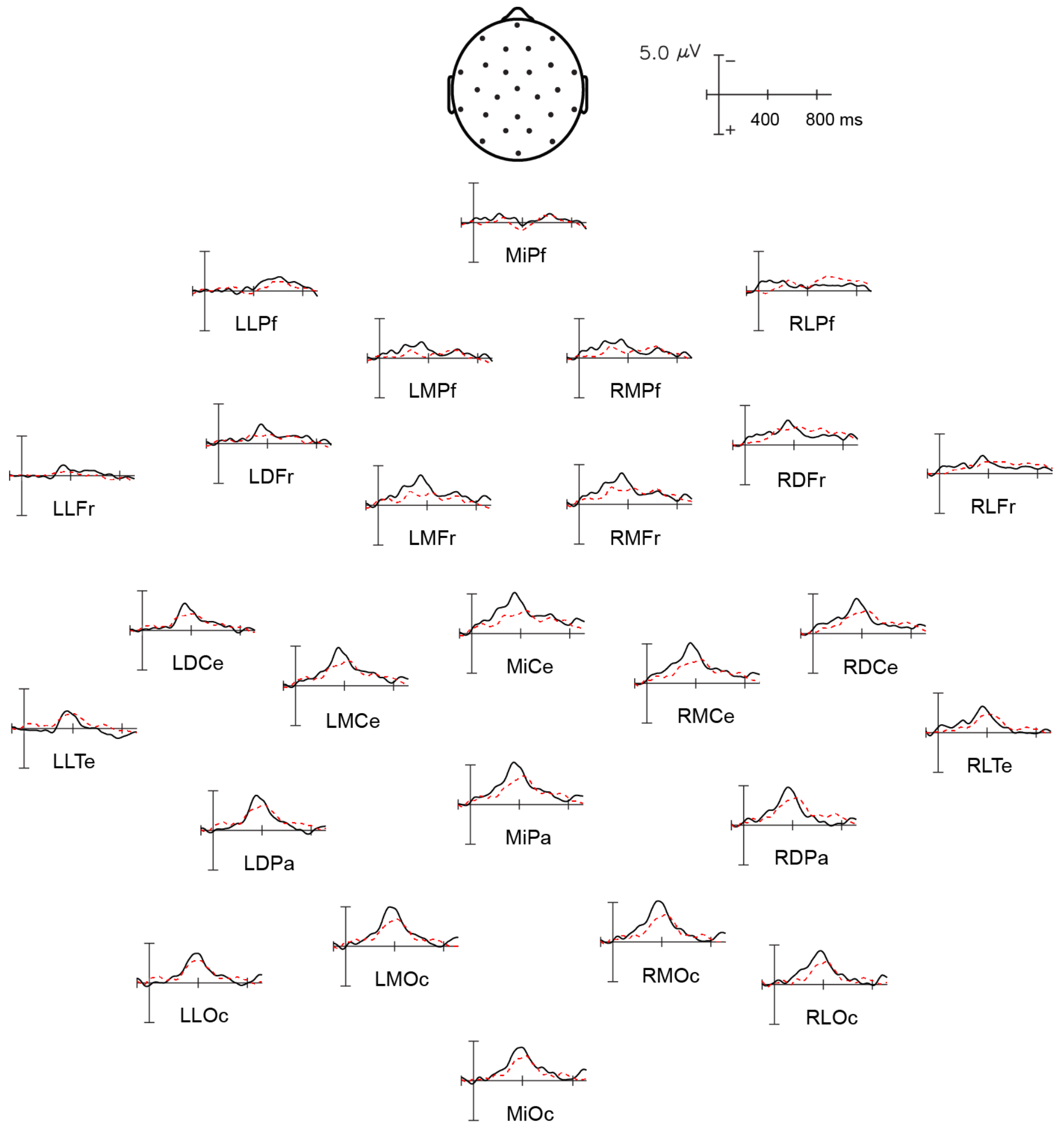

General Inconsistent minus General Consistent

Personal Inconsistent minus Personal Consistent 
Figure 3. Normalized Topographic Maps of General and Personal Difference Waves

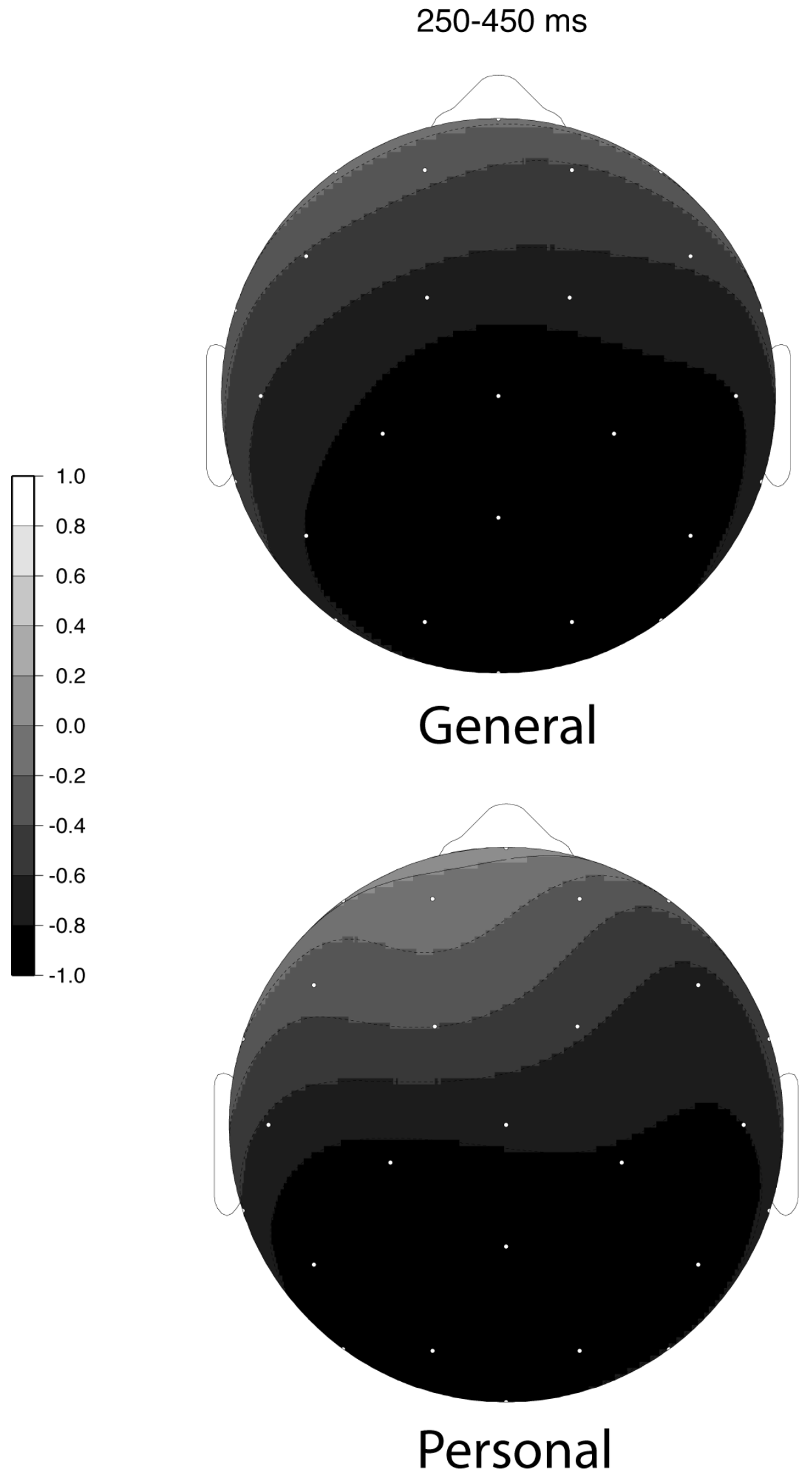

Note: The figure shows normalized (1 to -1) topographic maps of general and personal difference waves for the 250 to $450 \mathrm{~ms}$ timewindow. 


\section{Discussion}

Our study revealed that personal semantics, in the form of self-knowledge about personal preferences, manifests in modulations of the N400 component of the ERP. Moreover, we showed that this N400 response for personal preferences is similar in multiple ways to the N400 elicited in response to general knowledge. More specifically, we found that N400 responses for both general and personal preferences manifested similar timing and scalp distributions. These similarities are notable given that we carefully selected stimuli in order to ensure that we could clearly distinguish participants' personal preferences from their knowledge about general preferences. In particular, we used a participant's preferences as stimuli for the personal condition only if he or she viewed them as distinct from most people's preferences. Thus, our findings show that personal semantics elicits an N400 response that is similar in functional sensitivity, distribution, and latency, to that for general semantic information. This result suggests that personal preferences share important functional and neurobiological features with general semantics.

The only difference in the N400 response to the personal versus the general semantic condition was a tendency for smaller congruency effects for the personal preference sentences. One of the most important determinants of N400 amplitude in sentences is expectancy, often indexed by cloze probability (e.g., Wlotko \& Federmeier, 2012). We obtained cloze probability measures for our general preference statements, but there were no similar metrics available to assess expectation strength for the personal preference sentences. Here, we adopted the approach of asking participants to self-assess the strength of the personal preference they provided. There was no clear way to precisely align the cloze probability data with these self-ratings; we 
attempted to achieve roughly similar levels of expectation by choosing general preference sentences of moderate to high cloze and personal preference statements with moderate to high self-assessed preference. This approach seems to have allowed a reasonable degree of success, given that both sentence types yielded robust effects. The fact that effect sizes were slightly smaller in the personal condition suggests either that word expectancies for the congruent items in these sentences were not as strong as within the general condition or that incongruent items were less incongruent. It will be interesting for future work to use the N400 to determine what factors affect expectancy in the context of personal semantics - e.g., how preferences and their strength can be reliably assessed, whether (and, if so, how) preference is graded, and how stable preference strengths are over time.

Differences in the processing of personal semantics and general knowledge were most salient in a time-window after the N400, in the form of larger LPC responses to sentences targeting personal preferences. These results are perhaps most similar to the ones obtained in the Renoult et al. (2015) study, which found no differences in N400 amplitude to names of famous people associated with high and low levels of personal memories. Instead, the amount of personal episodic memories linked to a name modulated the later-occurring LPC, which is often associated with explicit recollection of contextual details (for reviews, see Rugg \& Curran, 2007; Voss \& Paller, 2008). Indeed, our results replicate previous studies, described in the introduction, that have found greater P300/LPC responses to self-relevant information. Based on the results from Renoult et al.'s (2015) study, one possible interpretation of our data is that participants may be retrieving personal episodic memories associated with their personal preferences. Given these findings, a relevant question is the extent to which access to information about personal preferences involves the retrieval of both acontextual and episodic components. Our study was 
not specifically designed to answer this question, but it is an important one for future research, as it may provide additional information about how semantic and episodic knowledge individually and jointly contribute to different domains of personal semantics.

Our findings may also speak to questions about the neural networks that underlie the access of personal semantics in the form of self-knowledge. Investigations into the neural sources of the N400 from a number of methods (e.g., magnetoencephalography, event-related optical signal, intracranial recordings) collectively point to sources in the medial temporal lobe, temporo-parietal junction, superior/middle temporal gyrus and some frontal areas (for a review, see Kutas \& Federmeier, 2011). Converging evidence from fMRI and studies of brain-damaged populations further suggests that this distributed network of brain areas is critical to semantic memory processes and storage. Our results therefore point to these brain regions as a common neural network supporting the access of general and personal semantics.

Although our findings suggest important similarities in how general and personal semantics are processed, caution is warranted in generalizing these findings to all aspects of selfrelated knowledge. The bulk of studies about self-knowledge in the neuropsychological literature (e.g., studies of amensic patients, etc.) have focused largely on personality traits (Klein \& Gangi, 2010), which could be processed differently from other types of information for reasons besides the fact that they constitute personal knowledge. Questions about the integrity of knowledge of personal preferences after the onset of a neuropathology has not been systematically studied. For example, many of the observations of intact knowledge of (pre-morbid) personal preferences among amnesic patients have been anecdotal in nature (Heilman \& Valenstein, 2011). Our work thus expands the pool of domains by which to study the nature and organization of selfknowledge and semantic memory. Moreover, it seems fruitful for future work to examine 
similarities and differences between the processing of self-knowledge and knowledge of individual people more generally, as ERP studies of person-specific processing have also reported both N400 (e.g.,Wiese \& Schweinberger, 2015) and LPC (Bartholow et al., 2001) effects.

In sum, theoretical views about personal semantics recognize that they come in a variety of forms (e.g., personal beliefs, personality traits, autobiographical facts) that differ in the extent to which they are associated with unique contextual details during encoding (e.g., perceptual, spatial, temporal information, etc.; similar to episodic memories) or are relatively abstract or concept-based (i.e., not associated with a specific encoding context; similar to general semantic memory) (Conway, 2005; Martinelli, Sperduti, \& Piolino, 2013). This distinction has been central to models describing the organization of personal semantic memory (Renoult et al., 2012). Our data suggest that, on this kind of continuum, knowledge of personal preferences is closer to general semantics and suggest that they share important functional and neurobiological features.

\section{Acknowledgements}

The authors are grateful to Karen Sixkiller for collecting the data. We also thank Priya Kandhadai for her assistance in creating the stimuli. This work was supported by a James S. McDonnell Foundation Scholar Award and NIH grant AG026308 to Kara D. Federmeier and a National Science Foundation SBE Postdoctoral Research Fellowship (SMA-1360732) to Jason C. Coronel. 


\section{References}

Amodio, D. M., \& Frith, C. D. (2006). Meeting of minds: the medial frontal cortex and social cognition. Nature Reviews Neuroscience, 7(4), 268-277. http://doi.org/10.1038/nrn1884

Bartholow, B. D., Fabiani, M., Gratton, G., \& Bettencourt, B. A. (2001). A psychophysiological examination of cognitive processing of and affective responses to social expectancy violations. Psychological Science, 12(3), 197-204. http://doi.org/10.1111/14679280.00336

Conway, M. A. (2005). Memory and the self. Journal of Memory and Language, 53(4), 594628. http://doi.org/10.1016/j.jml.2005.08.005

Federmeier, K. D., \& Kutas, M. (1999). A Rose by Any Other Name: Long-Term Memory Structure and Sentence Processing. Journal of Memory and Language, 41(4), 469-495. http://doi.org/10.1006/jmla.1999.2660

Federmeier, K. D., \& Laszlo, S. (2009). Chapter 1 Time for Meaning: Electrophysiology Provides Insights into the Dynamics of Representation and Processing in Semantic Memory. In B. H. Ross (Ed.), Psychology of Learning and Motivation (Vol. 51, pp. 144). Academic Press. Retrieved from http://www.sciencedirect.com/science/article/pii/S0079742109510018

Fields, E. C., \& Kuperberg, G. R. (2012). It's All About You: an ERP study of emotion and selfrelevance in discourse. NeuroImage, 62(1), 562-574. http://doi.org/10.1016/j.neuroimage.2012.05.003

Gillihan, S. J., \& Farah, M. J. (2005). Is self special? A critical review of evidence from experimental psychology and cognitive neuroscience. Psychological Bulletin, 131(1), 7697. http://doi.org/10.1037/0033-2909.131.1.76 
THE N400 AND PERSONAL SEMANTICS

Grilli, M. D., \& Verfaellie, M. (2014). Personal semantic memory: insights from neuropsychological research on amnesia. Neuropsychologia, 61, 56-64. http://doi.org/10.1016/j.neuropsychologia.2014.06.012

Hagoort, P., Hald, L., Bastiaansen, M., \& Petersson, K. M. (2004). Integration of Word Meaning and World Knowledge in Language Comprehension. Science, 304(5669), 438-441. http://doi.org/10.1126/science.1095455

Heatherton, T. F. (2011). Neuroscience of Self and Self-Regulation. Annual Review of Psychology, 62, 363-390. http://doi.org/10.1146/annurev.psych.121208.131616

Heatherton, T. F., Macrae, C. N., \& Kelley, W. M. (2004). What the Social Brain Sciences Can Tell Us About the Self. Current Directions in Psychological Science, 13(5), 190-193. http://doi.org/10.1111/j.0963-7214.2004.00305.x

Heilman, K. M., \& Valenstein, E. (2011). Clinical Neuropsychology. Oxford University Press.

Kelley, W. M., Macrae, C. N., Wyland, C. L., Caglar, S., Inati, S., \& Heatherton, T. F. (2002). Finding the self? An event-related fMRI study. Journal of Cognitive Neuroscience, 14(5), 785-794. http://doi.org/10.1162/08989290260138672

Klein, S. B., Chan, R. L., \& Loftus, J. (1999). Independence of Episodic and Semantic SelfKnowledge: The Case from Autism. Social Cognition, 17(4), 413-436. http://doi.org/10.1521/soco.1999.17.4.413

Klein, S. B., Cosmides, L., \& Costabile, K. A. (2003). Preserved Knowledge of Self in a Case of Alzheimer's Dementia. Social Cognition, 21(2), 157-165. http://doi.org/10.1521/soco.21.2.157.21317 
Klein, S. B., Cosmides, L., Costabile, K. A., \& Mei, L. (2002). Is there something special about the self? A neuropsychological case study. Journal of Research in Personality, 36(5), 490-506. http://doi.org/10.1016/S0092-6566(02)00001-6

Klein, S. B., \& Gangi, C. E. (2010). The multiplicity of self: neuropsychological evidence and its implications for the self as a construct in psychological research. Annals of the New York Academy of Sciences, 1191, 1-15. http://doi.org/10.1111/j.1749-6632.2010.05441.x

Kounios, J., \& Holcomb, P. J. (1992). Structure and process in semantic memory: evidence from event-related brain potentials and reaction times. Journal of Experimental Psychology. General, 121(4), 459-479.

Kuperberg, G. R., Sitnikova, T., Caplan, D., \& Holcomb, P. J. (2003). Electrophysiological distinctions in processing conceptual relationships within simple sentences. Brain Research. Cognitive Brain Research, 17(1), 117-129.

Kutas, M., \& Federmeier, K. D. (2011). Thirty years and counting: Finding meaning in the N400 component of the event related brain potential (ERP). Annual Review of Psychology, 62, 621-647. http://doi.org/10.1146/annurev.psych.093008.131123

Lau, E. F., Phillips, C., \& Poeppel, D. (2008). A cortical network for semantics: (de)constructing the N400. Nature Reviews. Neuroscience, 9(12), 920-933. http://doi.org/10.1038/nrn2532

Martinelli, P., Sperduti, M., \& Piolino, P. (2013). Neural substrates of the self-memory system: new insights from a meta-analysis. Human Brain Mapping, 34(7), 1515-1529. http://doi.org/10.1002/hbm.22008

Northoff, G., \& Bermpohl, F. (2004). Cortical midline structures and the self. Trends in Cognitive Sciences, 8(3), 102-107. http://doi.org/10.1016/j.tics.2004.01.004 
THE N400 AND PERSONAL SEMANTICS

Oldfield, R. C. (1971). The assessment and analysis of handedness: the Edinburgh inventory. Neuropsychologia, 9(1), 97-113.

Renoult, L., Davidson, P. S. R., Palombo, D. J., Moscovitch, M., \& Levine, B. (2012). Personal semantics: at the crossroads of semantic and episodic memory. Trends in Cognitive Sciences, 16(11), 550-558. http://doi.org/10.1016/j.tics.2012.09.003

Renoult, L., Davidson, P. S. R., Schmitz, E., Park, L., Campbell, K., Moscovitch, M., \& Levine, B. (2015). Autobiographically significant concepts: more episodic than semantic in nature? An electrophysiological investigation of overlapping types of memory. Journal of Cognitive Neuroscience, 27(1), 57-72.http://doi.org/10.1162/jocn_a_00689

Renoult, L., Tanguay, A., Beaudry, M., Tavakoli, P., Rabipour, S., Campbell, K., ... Davidson, P. S. R. (2015). Personal semantics: Is it distinct from episodic and semantic memory? An electrophysiological study of memory for autobiographical facts and repeated events in honor of Shlomo Bentin. Neuropsychologia. http://doi.org/10.1016/j.neuropsychologia.2015.08.013

Rogers, T. B., Kuiper, N. A., \& Kirker, W. S. (1977). Self-reference and the encoding of personal information. Journal of Personality and Social Psychology, 35(9), 677-688. http://doi.org/10.1037/0022-3514.35.9.677

Rugg, M. D., \& Curran, T. (2007). Event-related potentials and recognition memory. Trends in Cognitive Sciences, 11(6), 251-257. http://doi.org/10.1016/j.tics.2007.04.004

Symons, C. S., \& Johnson, B. T. (1997). The self-reference effect in memory: a meta-analysis. Psychological Bulletin, 121(3), 371-394. http://doi.org/10.1037/0033-2909.121.3.371

Taylor, W. (1953). Cloze procedure: A new tool for measuring readability. Journalism Quarterly, (30), 415-433. 
THE N400 AND PERSONAL SEMANTICS

Tulving, E. (1983). Elements of Episodic Memory. Clarendon Press.

Tulving, E. (2002). Episodic Memory: From Mind to Brain. Annual Review of Psychology, 53(1), 1-25. http://doi.org/10.1146/annurev.psych.53.100901.135114

Urbach, T. P., \& Kutas, M. (2010). Quantifiers more or less quantify online: ERP evidence for partial incremental interpretation. Journal of Memory and Language, 63(2), 158-179. http://doi.org/10.1016/j.jml.2010.03.008

Van Petten, C., \& Luka, B. J. (2006). Neural localization of semantic context effects in electromagnetic and hemodynamic studies. Brain and Language, 97(3), 279-293. http://doi.org/10.1016/j.bandl.2005.11.003

Voss, J. L., \& Paller, K. A. (2008). Brain substrates of implicit and explicit memory: The importance of concurrently acquired neural signals of both memory types. Neuropsychologia, 46(13), 3021-3029. http://doi.org/10.1016/j.neuropsychologia.2008.07.010

Wagner, D. D., Haxby, J. V., \& Heatherton, T. F. (2012). The Representation of Self and Person Knowledge in the Medial Prefrontal Cortex. Wiley Interdisciplinary Reviews. Cognitive Science, 3(4), 451-470. http://doi.org/10.1002/wcs.1183

Wiese, H., \& Schweinberger, S. R. (2015). Getting connected: Both associative and semantic links structure semantic memory for newly learned persons. Quarterly Journal of Experimental Psychology (2006), 68(11), 2131-2148. http://doi.org/10.1080/17470218.2015.1008526

Wlotko, E. W., \& Federmeier, K. D. (2012). Age-related changes in the impact of contextual strength on multiple aspects of sentence comprehension. Psychophysiology, 49(6), 770785. http://doi.org/10.1111/j.1469-8986.2012.01366.x 


\section{Footnotes:}

${ }^{1}$ On average, $63 \%$ of the participants' personal preferences overlapped with the target final word in the general consistent condition. Note that participants were given the opportunity to provide their first, secondary, and tertiary preferences. If any of their stated preferences (first, second, third) matched the target final word in the general consistent condition, this trial was counted as an "overlap". Our aim was to make sure that responses to the personal condition could not arise just due to general semantic knowledge, which is why we took care that the congruent personal preferences were dissociable from the general case. Given that N400 effects to general knowledge are well-established, we were not similarly worried about interpretational issues related to overlap with personal preferences for that condition. For the general inconsistent condition, we specifically avoided using sentences in which the target ending word matched any of the participants' stated preferences. 